Document downloaded from:

http://hdl.handle.net/10251/57095

This paper must be cited as:

Lluna Gil, E.; Santiago-Praderas, V.; Defez Garcia, B.; Dunai, L.; Peris Fajarnes, G. (2011). Velocity vector (3D) measurement for spherical objects using an electro-optical device. Measurement. 44(9):1723-1729. doi:10.1016/j.measurement.2011.07.006.

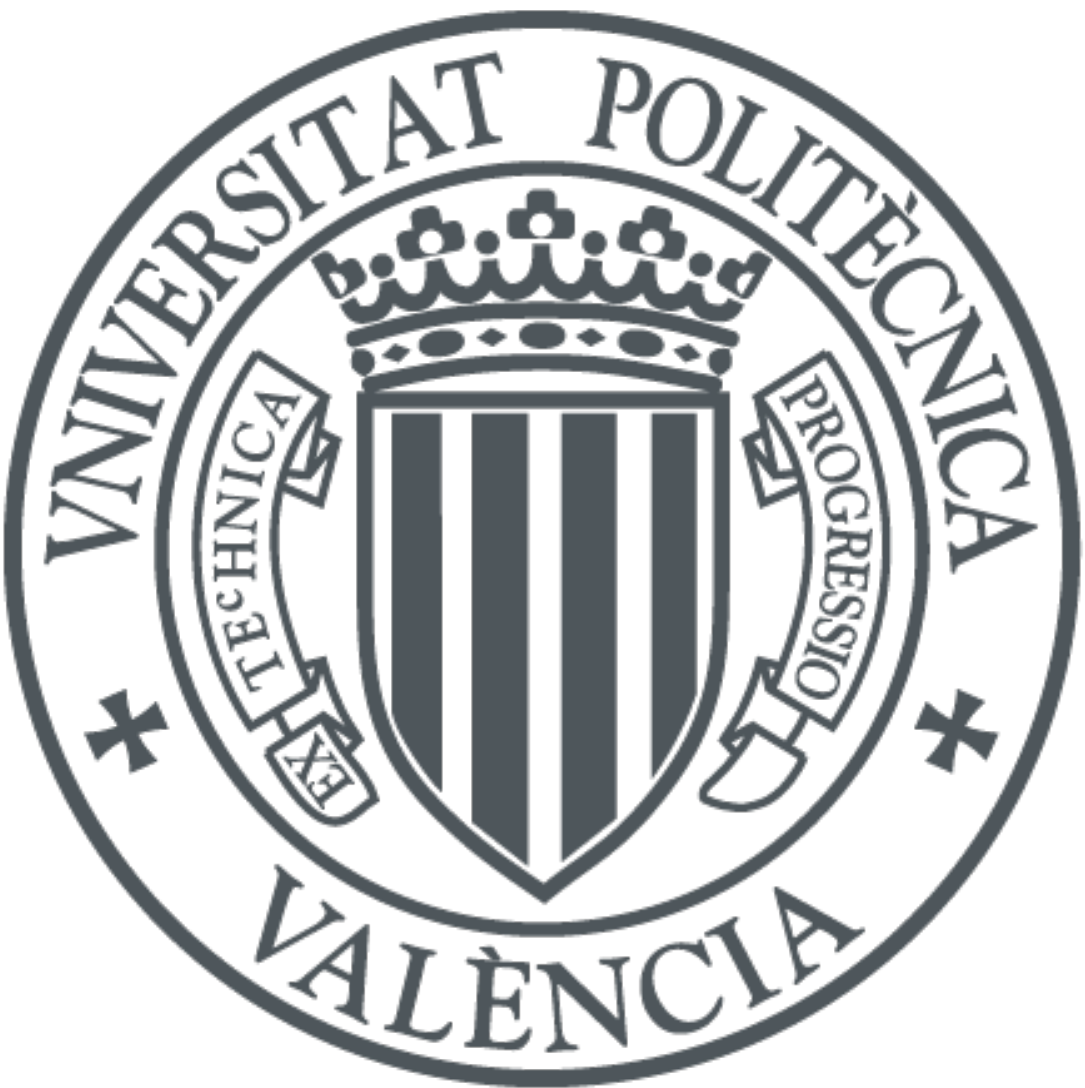

The final publication is available at

http://dx.doi.org/10.1016/j.measurement.2011.07.006

Copyright Elsevier

Additional Information 
Document downloaded from:

http://hdl.handle.net/10251/57095

This paper must be cited as:

Lluna Gil, E.; Santiago-Praderas, V.; Defez Garcia, B.; Dunai, L.; Peris Fajarnes, G. (2011). Velocity vector (3D) measurement for spherical objects using an electro-optical device. Measurement. 44(9):1723-1729. doi:10.1016/j.measurement.2011.07.006.

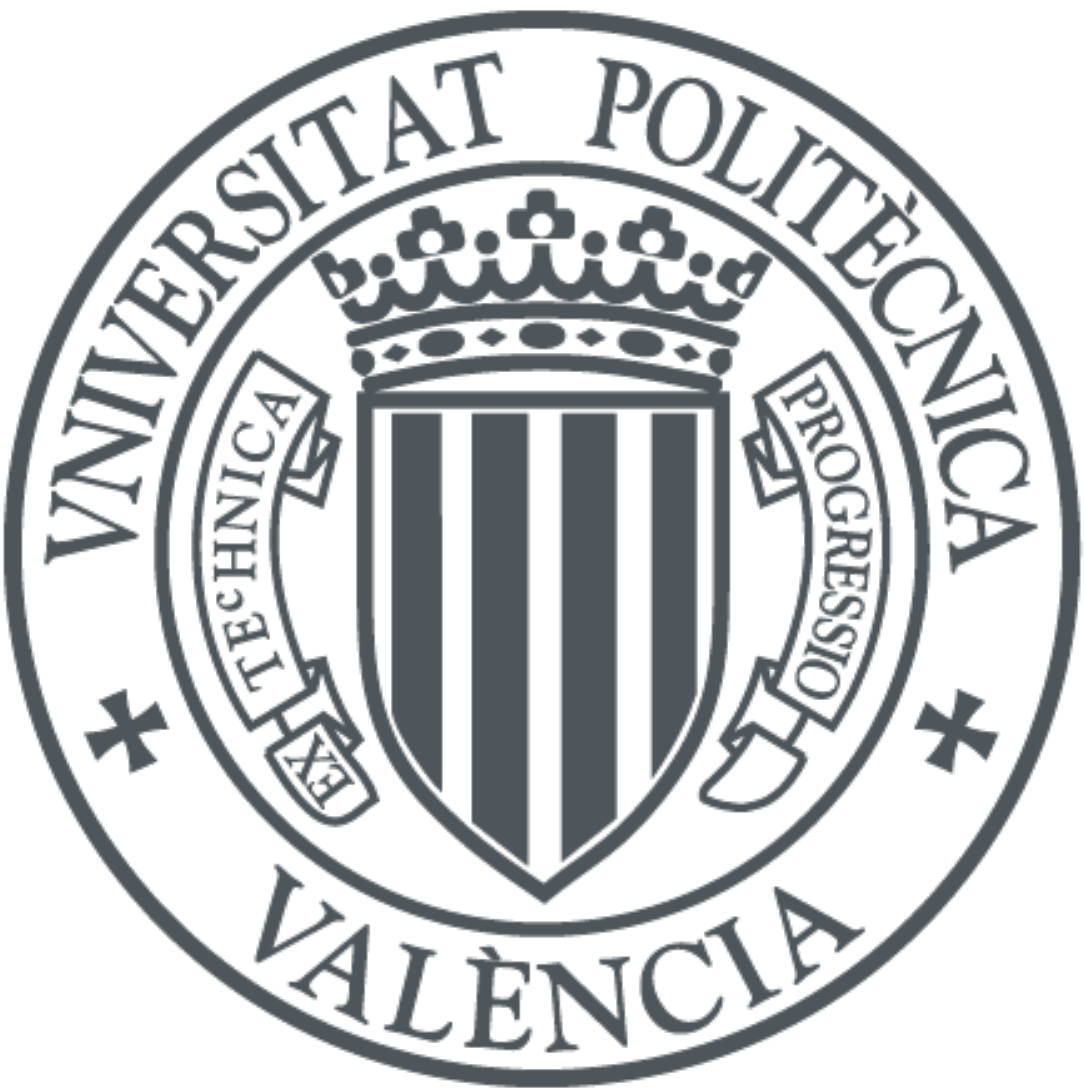

The final publication is available at

http://dx.doi.org/10.1016/j.measurement.2011.07.006

Copyright Elsevier

Additional Information 


\title{
Velocity vector (3D) measurement for spherical objects using an electro-optical device
}

\author{
E. Lluna, V. Santiago, B. Defez, L. Dunai, G. Peris-Fajarnes \\ edllugi@doctor.upv.es \\ Universidad Politécnica de Valencia \\ Centro de Investigación en Tecnologías Gráficas (CITG), Edificio-8L \\ Camino de Vera s/n, E-46022 Valencia, Spain
}

\begin{abstract}
The present paper describes a procedure to measure the velocity vector (3D) of a spherical object using an electro-optical device configured as a single large detection area optical barrier. The proposed procedure allows a measurement accuracy up to $0.1 \%$ in some cases and presents several advantages in relation to other measurement procedures like image processing, doppler-radar and some other electro-optical devices. The procedure is independent of the relative position of the measurement device in relation to the object trajectory. The fact of using a single optical barrier reduces the space required in the movement direction and increase the cases where the device can be used. A prototype has been built and tested.
\end{abstract}

Keywords: velocity measurement, optoelectronic, optical barrier, non contact measurement.

\section{Introduction}

There are many applications that require the measurement of some dynamical parameters for a moving object of known geometry. These applications include the training of sports using balls, ballistics and aeronautical ones. In particular, the velocity vector is the most important dynamical parameter. For small objects or in the case that it is not possible to install a measurement device on it, external procedures are required to measure the required parameters. There are several non contact measurement methods, some based on image processing $[1,2]$, using doppler-radar devices $[3,4,5]$ or any type of electro-optical device configured as optical barriers $[6,7,8]$. The methods based on image processing use high speed cameras to record the movement and extract the information of the time used by the object to fly across a known distance and then, the velocity is calculated. This procedure presents some drawbacks because it is not easy to perform the image recognition on real-time, it requires expensive high speed cameras and it is highly dependant on the position of the camera in relation to the object trajectory. Most of the methods use electro-optical devices because they have a lower cost than cameras or doppler-radar devices, moreover they allow to make instantaneous calculations. The typical setup used on ballistics $[9,10,11]$ maintain the problem of the dependency with the position of the measurement device in relation to the object trajectory and they only measure the velocity in one direction component.

The present work shows a method to measure the three components of the velocity vector $\left(v_{x}, v_{y}, v_{z}\right)$ using a single large detection area optical barrier $[12,13,14]$. The procedure does not depend on the relative position of the device in relation with the trajectory of the object. The independence of the position and the fact of using a large detection area device increases the cases in which the device can be used. The method allows real time calculation and, placing several optical barriers one behind the other at known separation distances, it is possible to measure the velocity in two known points and then calculate the acceleration.

This paper is structured as follows. Section 2 presents the theoretical model developed. Section 3 presents the validation of the proposed model using two procedures, a numerical simulation and real measurements using a prototype. Finally, section 4 presents the conclusions. 


\section{Theoretical Model}

A large detection area optical barrier consists on several beams (each composed by a transmitter and a receiver) forming a plane. Figure 1 shows the axis convention used. There is a fixed separation between consecutive beams $d_{\text {sep }}$.

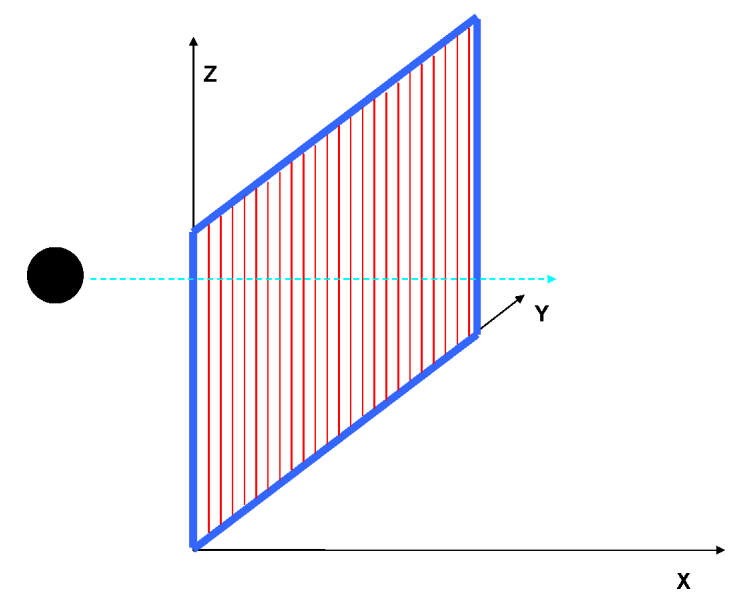

Figure 1: Axis convention in an optical barrier.

When an object passes through the optical barrier, the beams are cut and keep in this state until the object has passed. Let call sensor to each beam and activation to the fact that a beam is cut by an object. If the object diameter is known, measuring the time that a beam is activated, the object velocity in the $\mathrm{X}$ direction (perpendicular to the barrier plane) is easily calculated. But the time a detector is activated is not enough to calculate the velocity in the $\mathrm{Y}$ direction. If the object velocity has no $\mathrm{Y}$ component, that means that is passing through the detection area perpendicularly. Let be $\theta$ the angle of the object incidence from the $\mathrm{X}$ axis. Component $v_{y} \neq 0$ means $\theta \neq 0$.

An optical barrier can be represented by equation (1). The detectors are placed along the $\mathrm{Y}$ axis with a fixed $d_{s e p}$ distance between each adjacent ones.

$$
\left\{\begin{array}{l}
x=0 \\
y=n d_{\text {sep }} \quad n=0, \pm 1, \pm 2, \ldots
\end{array}\right.
$$

A spherical object is characterized by its center position and its radius $r$. If the object has a velocity $v=\left(v_{x}, v_{y}\right)$ (XY plane is considered) and departs from an initial point $\left(x_{0}, y_{0}\right)$ it is described by equation (2).

$$
\left(x-\left(x_{0}+v_{x} t\right)\right)^{2}+\left(y-\left(y_{0}+v_{y} t\right)\right)^{2}=r^{2}
$$

The time that the detectors of the optical barrier are activated and deactivated corresponds to the values of the equation system composed by equations (1) and (2).

$$
\begin{gathered}
t^{2}\left(v_{x}^{2}+v_{y}^{2}\right)+ \\
t\left(-2 x v_{x}-2 y v_{y}+2 x_{0} v_{x}+2 y_{0} v_{y}\right)+ \\
\left(x^{2}+y^{2}-2 x x_{0}-2 y y_{0}+x_{0}^{2}+y_{0}^{2}-r^{2}\right)=0
\end{gathered}
$$

Equation (4) and (5) is the solution and gives the activation and deactivation time for each detector that is affected by the object movement.

$$
\begin{gathered}
t=\frac{-B \pm \sqrt{B^{2}-4 A C}}{2 A} \\
\left\{\begin{array}{l}
A=\left(v_{x}^{2}+v_{y}^{2}\right) \\
B=-2 x v_{x}-2 y v_{y}+2 x_{0} v_{x}+2 y_{0} v_{y} \\
C=x^{2}+y^{2}-2 x x_{0}-2 y y_{0}+x_{0}^{2}+y_{0}^{2}-r^{2}
\end{array}\right.
\end{gathered}
$$


Using equation (4) for two cases, one with $v=1 \mathrm{~m} / \mathrm{s}$ and $\theta=0 \mathrm{rad}$ and a second one with $v=1 \mathrm{~m} / \mathrm{s}$ and $\theta=p i / 8 \mathrm{rad}$, values shown on table 1 and 2 are obtained. The column 'Middle' corresponds to the instant time the center of the object (middle point because the symetry of the object) passes through the detector and it is calculated as the center of the interval between activation and deactivation time.

\begin{tabular}{|c|c|c|c|c|}
\hline Detector & Act. (s) & Deact. (s) & Duration (s) & Middle (s) \\
\hline \hline 5 & 0.16 & 0.24 & 0.08 & 0.20 \\
\hline 4 & 0.10 & 0.30 & 0.19 & 0.20 \\
\hline 3 & 0.09 & 0.31 & 0.22 & 0.20 \\
\hline 2 & 0.10 & 0.30 & 0.19 & 0.20 \\
\hline 1 & 0.16 & 0.24 & 0.08 & 0.20 \\
\hline
\end{tabular}

Table 1: Activation and deactivation time for $v=1 \mathrm{~m} / \mathrm{s}$ and $\theta=0$.

\begin{tabular}{|c|c|c|c|c|}
\hline Detector & Act. (s) & Deact. (s) & Duration (s) & Middle (s) \\
\hline \hline 4 & 0.11 & 0.26 & 0.15 & 0.19 \\
\hline 3 & 0.10 & 0.31 & 0.21 & 0.20 \\
\hline 2 & 0.12 & 0.33 & 0.21 & 0.22 \\
\hline 1 & 0.15 & 0.33 & 0.18 & 0.24 \\
\hline
\end{tabular}

Table 2: Activation and deactivation time for $v=1 \mathrm{~m} / \mathrm{s}$ and $\theta=p i / 8$.

The maximum time a detector is activated is the same in both cases. This is because the object symmetry. The maximum time always corresponds to the diameter transit and in both cases is the same. The difference in both cases is on the time the detectors start being activated. If the object has an incidence angle (the trajectory is not perpendicular to the detectors plane) the time a detector starts the detection is delayed or advanced with respect to the perpendicular case. So the time difference between the activation of consecutive detectors gives information about the incidence angle.

Taking the symmetry axis that passes through the middle of the object, its time correspond to the 'Middle' column. When the object has a perpendicular trajectory, all the detectors are activated at the same time (by the line of centers). If the object has a trajectory with certain angle, the instant a detector is activated in relation to other changes. Figure 2 shows the geometry in the instant a detector $(\# 1)$ is activated. To activate the second detector $(\# 2)$ the object must travel de distance $d$. This distance is $v t_{12}$, where $t_{12}$ is the time difference between the activation of two consecutive detectors and $v$ the velocity in the direction of the movement.

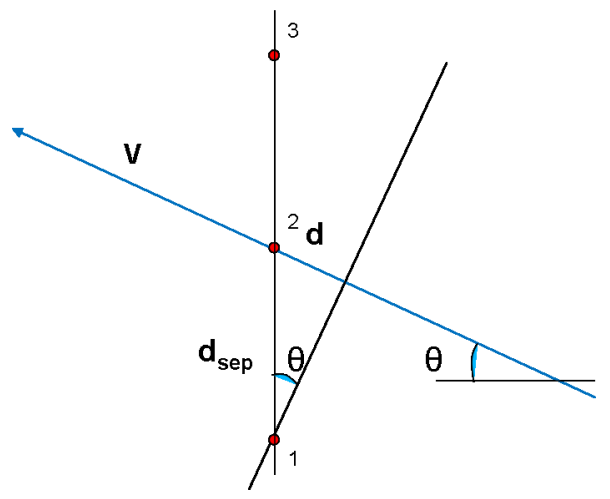

Figure 2: Detector activation geometry

The velocity in the movement direction is given by (6) where $d_{\text {diam }}$ is the diameter of the object and $t_{\text {max }}$ is the maximum time a detector is activated and corresponds, because of the symetry of the object, to the diameter. 


$$
v=\frac{d_{\text {diam }}}{t_{\max }}
$$

The angle of incidence $\theta$ is, from the geometry of figure 2 :

$$
\begin{gathered}
\sin \theta=\frac{d}{d_{\text {sep }}}=\frac{v t_{12}}{d_{\text {sep }}}=\frac{\left(d_{\text {diam }} / t_{\text {max }}\right) t_{12}}{d_{\text {sep }}}=\frac{d_{\text {diam }} t_{12}}{t_{\text {max }} d_{\text {sep }}} \\
\theta=\arcsin \left(\frac{d_{\text {diam }} t_{12}}{t_{\text {max }} d_{\text {sep }}}\right)
\end{gathered}
$$

With the values of $v$ and $\theta$ the values of $v_{x}$ and $v_{y}$ are obtained using (9) and (10).

$$
\begin{aligned}
& v_{x}=v \cos \theta \\
& v_{y}=v \sin \theta
\end{aligned}
$$

Each pair of detectors activated by the moving object gives a velocity value because the calculation is based on time differences between consecutive detectors. $n-1$ velocity values are obtained and the mean value is taken. An optical barrier with a line of detectors (along one axis), for example on the Y axis, gives two velocity components, $v_{x}$ and $v_{y}$. To obtain the third component, a second line of detectors, along the $\mathrm{Z}$ axis, is needed. This second line of detectors will provide, again the $v_{x}$ component and the $v_{z}$ one. Both $v_{x}$ velocities must be the same. The final velocity is then given by (11):

$$
v=\sqrt{v_{x}^{2}+v_{y}^{2}+v_{z}^{2}}
$$

\subsection{Error sources}

The values of $v_{x}$ and $v_{y}$ depend on the calculated values of $v$ and $\theta$. The error of $v$ and $\theta$ depends on the error in $d_{\text {diam }}, t_{\text {max }}, t_{12} \cdot d_{\text {sep }}$ is considered with no error. The absolute error values for the time magnitudes $\left(\varepsilon_{t m a x}\right.$ and $\left.\varepsilon_{t 12}\right)$ are determined by the time response of the optical device and the electronic circuitry used on the real measurement device. There is an error in the $d_{\text {diam }}$ value because a finite number of detector are used (it is not a continuous barrier) and there is a gap between them. Figure 3 shows on the left the best case, when the diameter of the object is aligned with a detector. On the right the worst case is shown. The diameter of the object passes through the gap between detectors, so the time used to calculate the speed with the known value of the diameter does not really correspond to the real diameter transit.
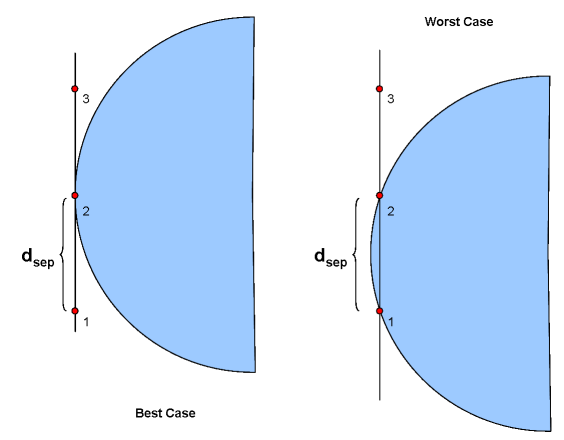

Figure 3: Error caused by the gap between detectors

Figure 4 shows the distance $r_{\text {err }}$ that corresponds to the error in the radius. The value is calculated from the rectangle formed by $d_{e r r}, d_{s e p} / 2$ and $r$. Equation (12) gives the $r_{e r r}$ value and (13) and (14) gives the absolute and relative error for $d_{\text {diam }}$. 


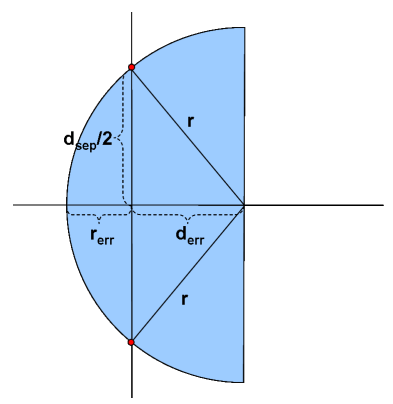

Figure 4: Diameter error in the worst case

$$
\begin{gathered}
r_{\text {err }}=r-d_{\text {err }}=r-\sqrt{r^{2}-\left(\frac{d_{\text {sep }}}{2}\right)^{2}} \\
\varepsilon_{\text {diam }}=2 r_{\text {err }} \\
\varepsilon_{\text {diam }}(\%)=\frac{\varepsilon_{\text {diam }}}{2 r} 100
\end{gathered}
$$

The error for an object of a fixed radio depends on the relation between $d_{s e p}$ and $r$. As the ratio $d_{\text {sep }} / r$ increases, the error also increases because $r_{\text {err }}$ is increased.

Table 3 shows the error for the object diameter $\left(\varepsilon_{\text {diam }}\right)$ for an object of a given diameter $(r=0.10 \mathrm{~m})$ for several values of $d_{\text {sep }}$. In the case that $d_{\text {sep }}$ value is $0.01 \mathrm{~m}$ the error is as low as $0.11 \%$.

\begin{tabular}{|c|c|c|}
\hline$d_{\text {sep }}$ & Abs.Err. $(\mathrm{m})$ & Rel.Err $(\%)$ \\
\hline \hline 0.00 & 0.0000 & 0.00 \\
\hline 0.01 & 0.0002 & 0.11 \\
\hline 0.02 & 0.0009 & 0.43 \\
\hline 0.03 & 0.0021 & 0.97 \\
\hline 0.04 & 0.0037 & 1.72 \\
\hline 0.05 & 0.0059 & 2.70 \\
\hline 0.06 & 0.0085 & 3.92 \\
\hline
\end{tabular}

Table 3: Error due to the distance among detectors.

Regarding the minimum number of detectors required, only two are enough to calculate the velocity but, because the error produced by the gap between detectors, if the diameter of the object is similar to this gap, the situation is similar to the one in which the gap is very high. So the number of required detectors determine the measure error.

The object diameter is considered as a known parameter but it can be calculated based on the number of activated detectors. In this case, the $\varepsilon_{\text {diam }}$ is incremented in $d_{\text {sep }} / 2$.

Because the method applies some calculations based on the time magnitudes, that are the real ones directly measured, the uncertainly of the calculated values is given by the error propagation theory.

\section{Testing the model}

Two procedures have been used to validate the theoretical model described on section 2 . The first method uses a numerical simulation applying the obtained equations. The second method uses a prototype of optical barrier to measure actual moving objects and compares the calculated velocity with the real one. 


\subsection{Simulation}

A Matlab simulation has been developed to test the model. Using equations (1) and (2) to calculate the time a beam is cut and released for an object with a known velocity vector. The initial velocity vector is reconstructed from the time value calculated and then compared with the initial value. The simulation allows to configure the best or worst case situation. The error is obtained comparing both values. The following Matlab code calculates $v$ and $\theta$ from the time values.

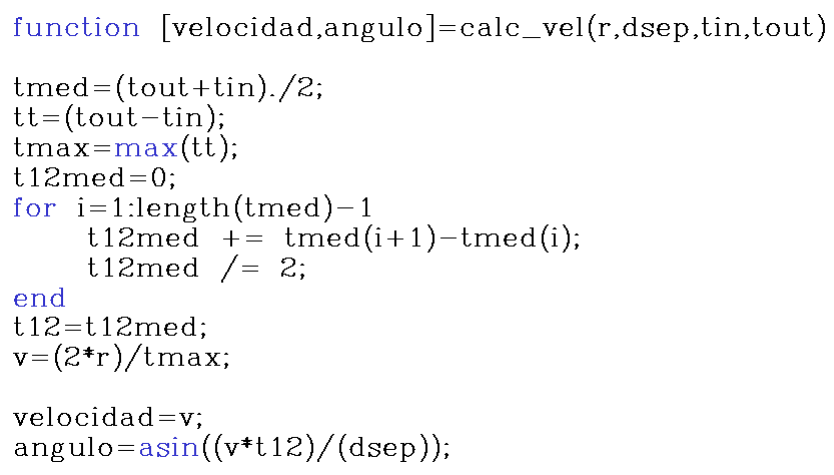

Figure 5: Matlab code to calculate $v$ and $\theta$.

The function receives the object diameter $r$, the distance between a pair of detectors $d_{\text {sep }}$, a list with the time the detectors have been cut $t_{\text {in }}$ and a list with the time the detectors have been released $t_{\text {out }}$.

\subsubsection{Simulation results}

Several cases have been tested using the simulation. The difference between the best and worst cases has been checked for an object that passes through the optical barrier with a perpendicular trajectory using $d_{\text {sep }}=0.05$ $\mathrm{m}$. In the best case the diameter was aligned with a beam and in the worst case, the diameter passes through the middle of the gap. In the best case the error is negligible and in the worst case is $2.78 \%$. This agrees with the expected value given by the equation 14 for the given $d_{s e p}$ value.

Several simulations have been executed for different incidence angles (from 0 to 60 degrees) using three different velocities. A low one of $1 \mathrm{~m} / \mathrm{s}$, a high speed of $70 \mathrm{~m} / \mathrm{s}$ in the range of the ones used in sports, and an intermediate value of $30 \mathrm{~m} / \mathrm{s}$. The results obtained using two different $d_{s e p}$ values and in the best and worst cases are shown in table 4 .

\begin{tabular}{|c|c|c|c|}
\hline$v$ & $d_{\text {sep }}$ & Case & $\varepsilon(\%)$ \\
\hline \hline 1 & 0.05 & best & 0 \\
\hline 1 & 0.01 & best & 0 \\
\hline 30 & 0.05 & best & $0\left(<1 \times 10^{-8}\right)$ \\
\hline 30 & 0.01 & best & $0\left(<1 \times 10^{-8}\right)$ \\
\hline 70 & 0.05 & best & $0\left(<1 \times 10^{-8}\right)$ \\
\hline 70 & 0.01 & best & $0\left(<1 \times 10^{-8}\right)$ \\
\hline 1 & 0.05 & worst & 2.7 \\
\hline 1 & 0.01 & worst & 0.1 \\
\hline 30 & 0.05 & worst & 2.7 \\
\hline 30 & 0.01 & worst & 0.1 \\
\hline 70 & 0.05 & worst & 2.7 \\
\hline 70 & 0.01 & worst & 0.1 \\
\hline
\end{tabular}

Table 4: Simulation results.

In the best case, the model gives the right velocity value without error (in the order of $1 e-8$ ) produced by the calculation rounds. In the worst case, the obtained errors are in the range shown in table 3 . For low values 
of $d_{\text {sep }}$ the value is as low as $0.1 \%$ and gets increased as $d_{\text {sep }}$ is increased reaching $2.7 \%$ in the case $d_{\text {sep }}=0.05$ $\mathrm{m}$.

\subsection{Experimental test}

To measure the three velocity components the optical barrier needs two lines of detectors, one along the Y axis to get the $v_{x}$ and $v_{y}$ components and the second along the $\mathrm{Z}$ axis to get the $v_{x}$ and $v_{z}$ components. The optical barrier measures the instant each detector is activated and deactivated and the relative time among beams.

The following subsections describe the developed hardware and software for the optical barrier.

\subsubsection{Prototype}

Figure 6 shows the basic hardware structure for the developed optical barrier. There are a set of emitters and receivers and a control $\mathrm{CPU}$. This $\mathrm{CPU}$ is connected to an external computer using a RS232/USB port. The barrier also includes an external synchronization line to synchronize measures with other optical barrier.

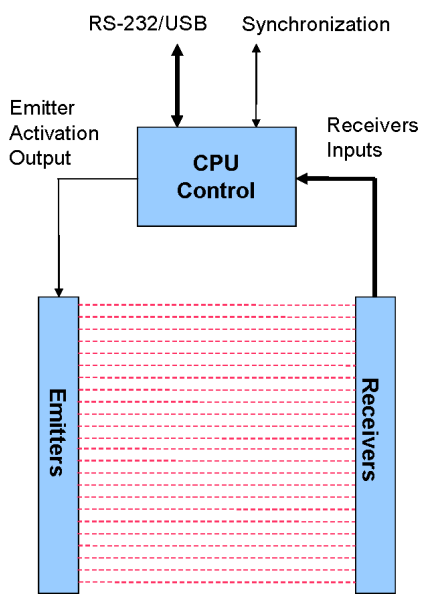

Figure 6: Optical barrier hardware blocks diagram.

Basic barrier parameters are $d_{\text {sep }}=0.05 \mathrm{~m}$ and $\varepsilon_{\text {time }}=0.0002 \mathrm{~s}$.

The emitters are lasers Z-Bolt ACC [15]. The main characteristics are Class IIIa, wavelength of $650 \mathrm{~nm}$ and power $<5 \mathrm{~mW}$. The receiver are phototransistors Vishay BPV-11 [16]. The main characteristics are sensibility in all the visible spectrum, and activation and deactivation times in the order of $5 \mu s$. The CPU used is the microcontroller PIC18F452 from Microchip [17].

Figure 7 shows the schematics for the emitter and receiver blocks. The ACT_LASERS line from the CPU activates/deactivates a relay that connects the power supply of the emitters. The collector of the phototransistors (lines Vo1... VoN) are connected to digital inputs in the CPU. The schema shown in Figure 7 is duplicated, one for each detector lines (horizontal and vertical). 

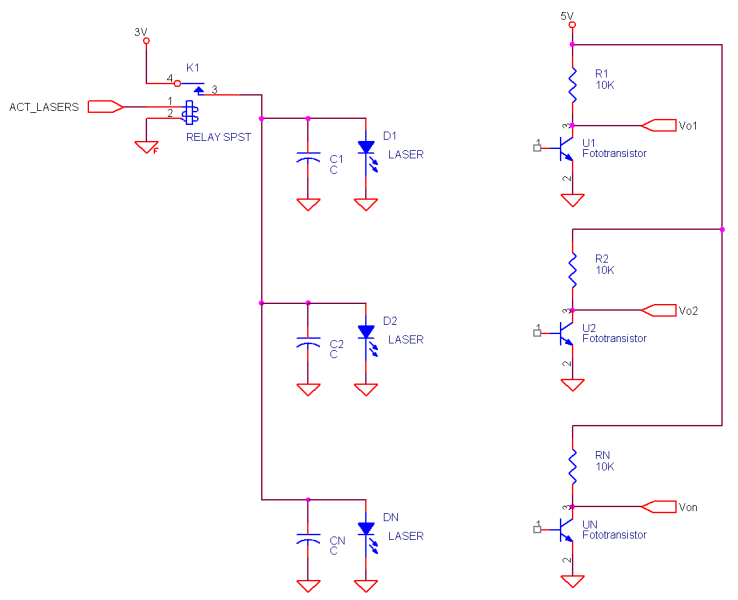

Figure 7: Emitters and Receivers block schematics.

To avoid the effect of the ambient light in the receivers a novel approach have been used. Instead of using the common procedure of modulating the beam, because the delays introduced by the demodulation, the phototransistors are placed inside a small pinhole cameras. The pinhole camera reduces the light that reaches the sensor but allows the beam to pass through the hole.

An important component of the optical barrier is the software. Two different softwares have been developed:

- The firmware that runs on the microcontroller CPU in the optical barrier.

- The software that runs on a external computer.

The firmware is responsible to activate/deactivate lasers and to measure the activation and deactivation times. A communication protocol has been defined to send commands to execute actions and retrieve the measured data. The sofware that runs on a external computer is responsible of the user interface and the calculation and it gets connected to the barrier through a RS232/USB interface. It sends the commands to the optical barrier to retrieve the measured data and performs the velocity calculation with the received information. The application handles up to two optical barriers. Figure 8 shows the main application window.

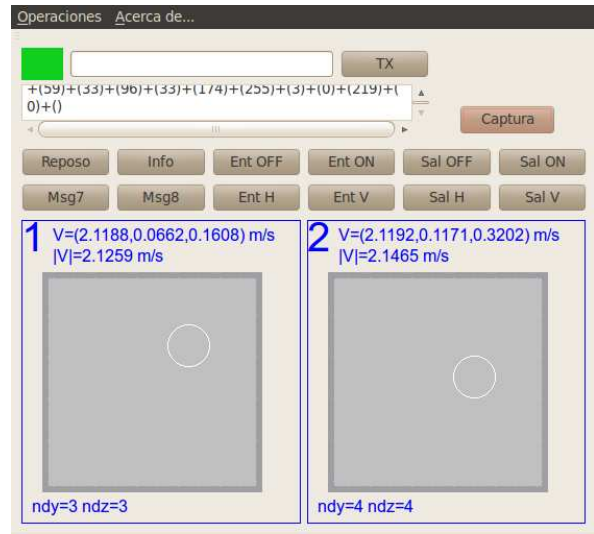

Figure 8: Control computer application.

\subsubsection{Prototype results}

To validate the method the velocity value measured by an optical barrier is compared with the value of the velocity calculated with other method. As second method, the velocity is calculated measuring the time used 
by the object to fly between two points at a fixed known distance. This second method gives the mean value of the velocity component in the $\mathrm{X}$ axis in the considered space. Let call it $v_{x 12}$. Two optical barrier are used, one on each of the points, to give the time instants to use in the second method calculation and the velocity vector in each point. From the velocity vector in each point, the mean value for the velocity component in the $\mathrm{X}$ axis can be calculated as $v_{x}=\left(v_{x 1}+v_{x 2}\right) / 2$ when $v_{x 1}$ and $v_{x 2}$ are the $\mathrm{X}$ components for the velocity values obtained in each of the optical barriers. If the distance between the optical barriers is $d_{f}$ and the times the object cut the barrier are $t_{i n 1}$ and $t_{i n 2}$, the velocity value for the second method is: $v_{x 12}=d_{f} /\left(t_{i n 2}-t_{i n 1}\right) . v_{x 12}$ and $v_{x}$ values must be the same.

Two optical barriers, like the ones described in 3.2.1, with $d_{f}=1 \mathrm{~m}$ were used. Table 5 shows some velocity measures for low velocity range. The velocities are in $\mathrm{m} / \mathrm{s}$ and the time values in $\mathrm{s}$. Table 6 shows the error values.

\begin{tabular}{|c|c|c|c|c|c|c|}
\hline Test & $v_{x 1}$ & $v_{x 2}$ & $v_{x}$ & $t_{i n 1}$ & $t_{i n 2}$ & $v_{x 12}$ \\
\hline \hline 1 & 4.40 & 4.34 & 4.37 & 1.67 & 1.90 & 4.48 \\
\hline 2 & 6.36 & 6.28 & 6.32 & 1.61 & 1.76 & 6.49 \\
\hline 3 & 8.24 & 8.08 & 8.16 & 1.42 & 1.53 & 8.39 \\
\hline 4 & 9.39 & 9.38 & 9.39 & 1.52 & 1.62 & 9.52 \\
\hline
\end{tabular}

Table 5: Prototypes results for velocity.

\begin{tabular}{|c|c|c|}
\hline Test & $\varepsilon$ & $\varepsilon(\%)$ \\
\hline \hline 1 & 0.12 & 2.56 \\
\hline 2 & 0.16 & 2.53 \\
\hline 3 & 0.23 & 2.72 \\
\hline 4 & 0.14 & 1.46 \\
\hline
\end{tabular}

Table 6: Errors for the measured values.

The difference $(\varepsilon(\%))$ is in the expected range because of the error introduced by a $d_{\text {sep }}=0.05 \mathrm{~m}$.

\section{Conclusions}

A procedure to measure the velocity vector $\left(v_{x}, v_{y}, v_{z}\right)$ of an object with known geometry using a unique large detection area optical barrier has been presented. The procedure introduces advantages in comparison with other procedures to measure the velocity vector of moving objects. If a non-continuous barrier is used, the separation between the detectors is the main factor for the measurement error. An error of less than $0.1 \%$ can be achieved with a separation of $1 \mathrm{~cm}$. Using a single optical barrier reduces the required space in the movement direction, increasing the cases the device can be used. The method has been tested with spherical objects, in particular soccer balls, but the method can be easily extended to other geometries. In relation with the design of the optical barrier, a new approach has been used to isolate the detector to the ambient light based on small pinhole cameras.

\section{References}

[1] John Eric Goff, Matt J. Carre. Trajectory analysis of a soccer ball. American Journal of Physics, Vol 77(11), November 2009.

[2] Allyson Beuhler, Francisco Castro, Anthony Polak. CMOS Camera with Integral Laser Ranging and Velocity Measurement". US Patent 6860350B2, March 2005.

[3] Yair Alon, Agoura Calif. Doppler Radar Method and Apparatus for Measuring Projectile's muzzly velocity". US Patent 1989. 
[4] Peter P. Toulios, Kenneth D. Hartman. Doppler Type Projectile Velocity Measurement and Communication Apparatus and Methods. US Patent 4282989, Aug 1981.

[5] M.Scheneider, D.Eckenfels, S.Nezirevic. Doppler-radar: A Possibility to Monitor Projectile Dynamics in Rail Guns. IEEE Transactions on Magnetics, Vol. 39, No.1, Jan 2003.

[6] M. Sanchez-Pana, M.Y. Fernandez, R. Zaera. Cost-effective optoelectronic system to measure the projectile velocity in high-velocity impact testing of aircraft and spacecraft structural elements. Optical Engineering. Vol.46(5), May 2007.

[7] Amandeep Kaur, Renu Vig, Randhir Bhatnagar. Study of Different Measurement Systems and Design Circuitry with Intensity Modulated Measuring the Velocity of Projectile. International Journal of Electronic Engineering Research. Vol. 1, No.2, 2009. pp 101-108.

[8] Václav Ricny, Jiri Mikulec. Measuring Flying Object Velocity with CCD Sensors. IEEE AES Systems Magazine, June 1994. pp 3-6.

[9] S.T.Lu, C.Chou, M.C. Lee, Y.P.Wu. Electro-optical target system for position and seed measurement. IEE Proceedings-A. Vol 140, No.4, July 1993. pp 252-256.

[10] G.S.Gill, Amod Kumar. Velocity Measurement System for Small Caliber Projectiles. WCE 2008. Vol.1 . July 2-4. London, UK.

[11] Yu Jiyan, Wang Xiaoming, Li Yongxin. Velocity and Position Measurement for Projectile Using Double Optical Detectors and Reflectors. IEEE 2009.

[12] Eldar Musayev. Laser-based large detection area speed measurement methods and systems. Optics and Lasers in Engineering. Vol 45, 2007, pp 1049-1054.

[13] Eldar Musa. Laser-based light barrier. Applied Optics. Vol 47, No. 19, Jul 2008, pp 3415-3422.

[14] Eldar Musa, Mutlu Demirer. Laser-based light barrier having a rectangular detection area. Optics and Lasers in Engineering. Vol 48, 2010, pp 435-440.

[15] Z-Bolt. ACC-5 Laser Module Data Sheet.

[16] Vishay. BPV11 Silicon NPN Phototransistor Data Sheet.

[17] Microchip. PIC18F452 Datasheet. www.microchip.com. 


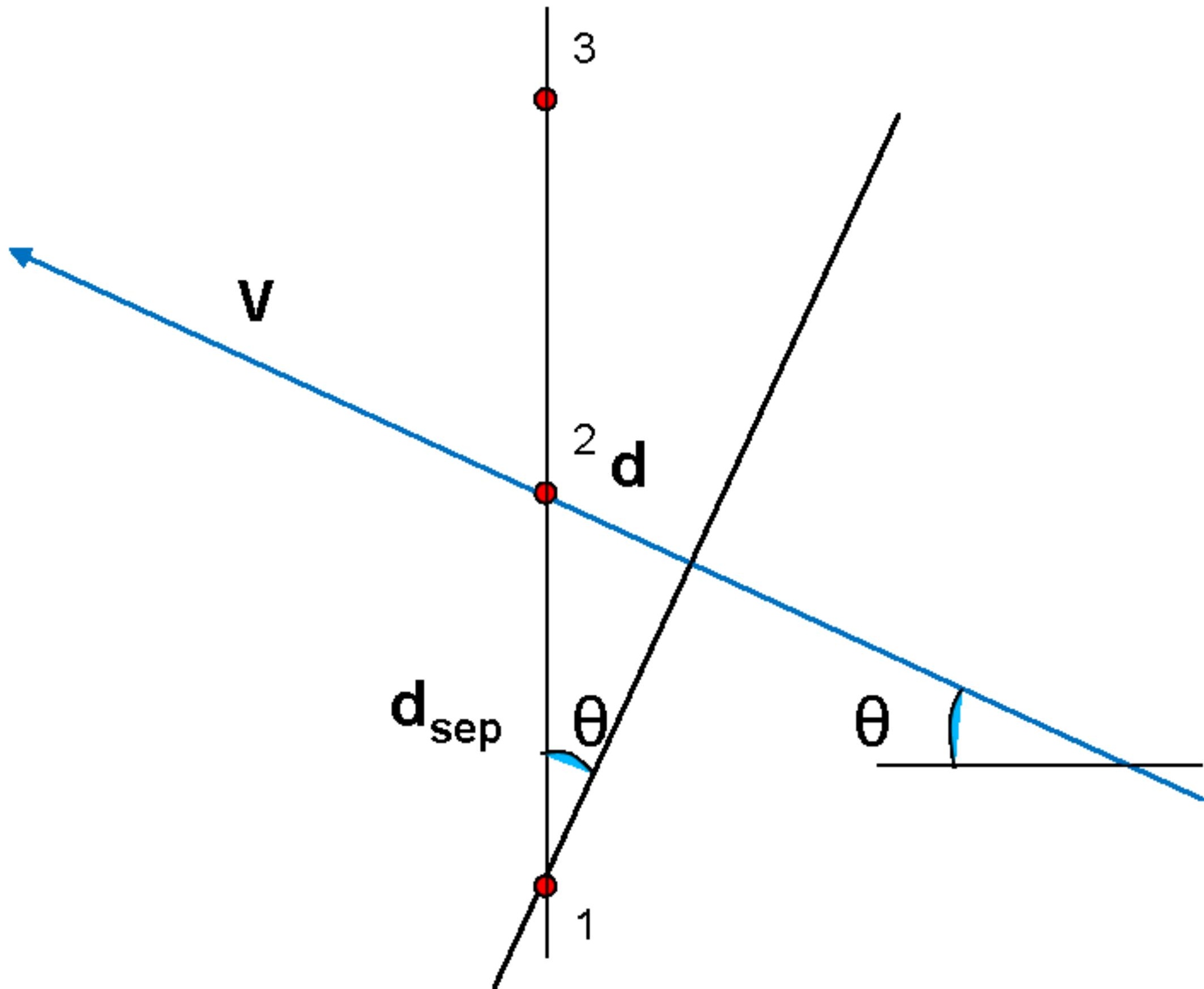




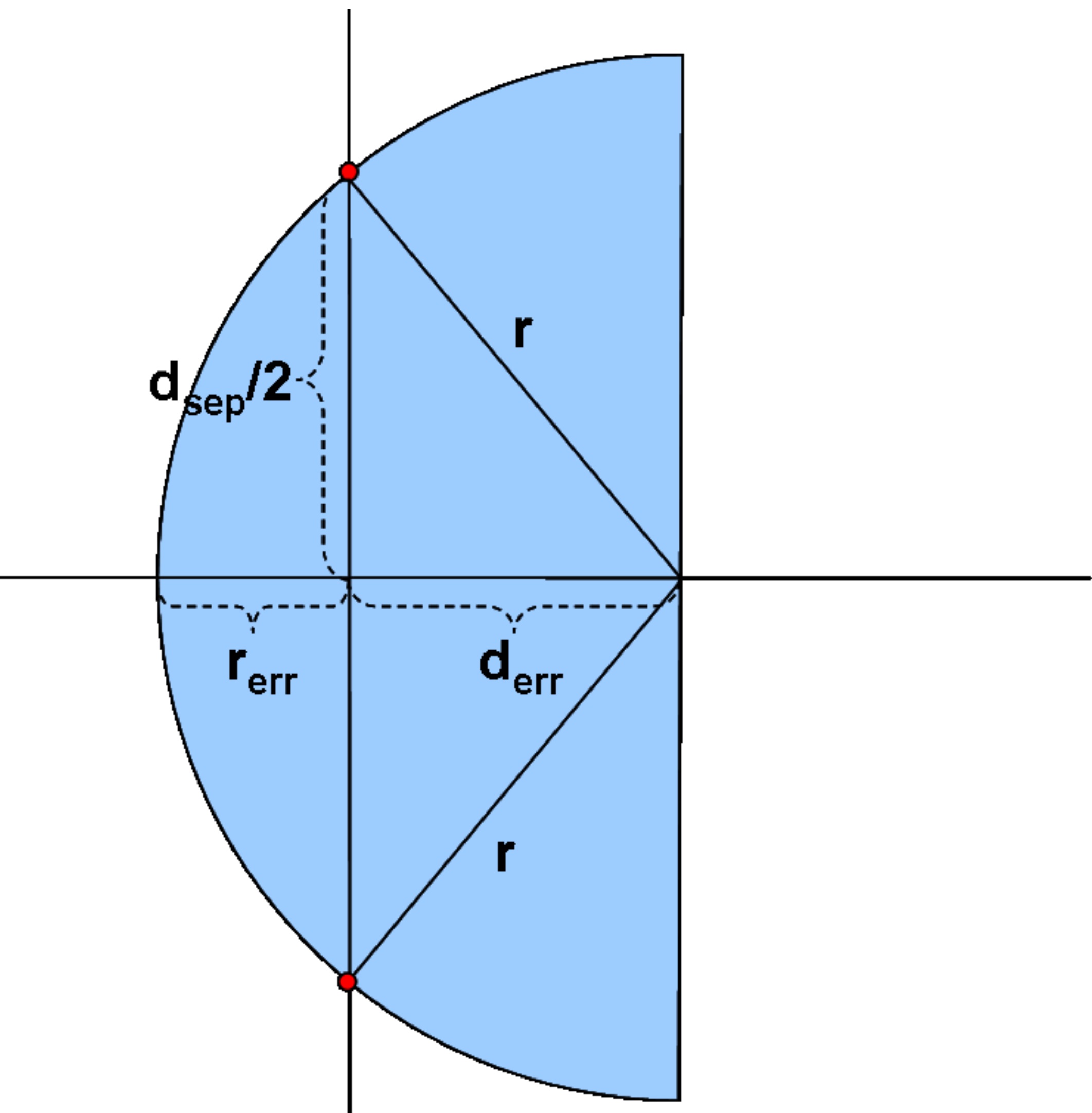


function [velocidad,angulo]=calc_vel(r,dsep,tin,tout)

tmed $=($ tout + tin $) \cdot / 2$

$\mathrm{tt}=($ tout $-\mathrm{tin})$

$\mathrm{tmax}=\max (\mathrm{tt})$

$\mathrm{t} 12 \mathrm{med}=0$

for $\mathrm{i}=1$ :length(tmed) -1

$t 12 \operatorname{med}+=t \operatorname{med}(i+1)-t \operatorname{med}(i)$;

t12med / = 2;

end

$\mathrm{t} 12=\mathrm{t} 12 \mathrm{med}$

$\mathrm{v}=(2 * \mathrm{r}) / \mathrm{t} \max$

velocidad $=\mathrm{v}$;

angulo $=\operatorname{asin}\left(\left(v^{*}+12\right) /(\right.$ dsep $\left.)\right)$; 


\section{RS-232/USB Synchronization}

\section{Emitter Activation Output}

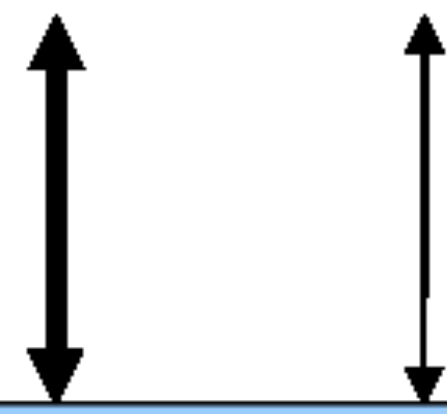

\section{CPU Control}

Receivers

Inputs

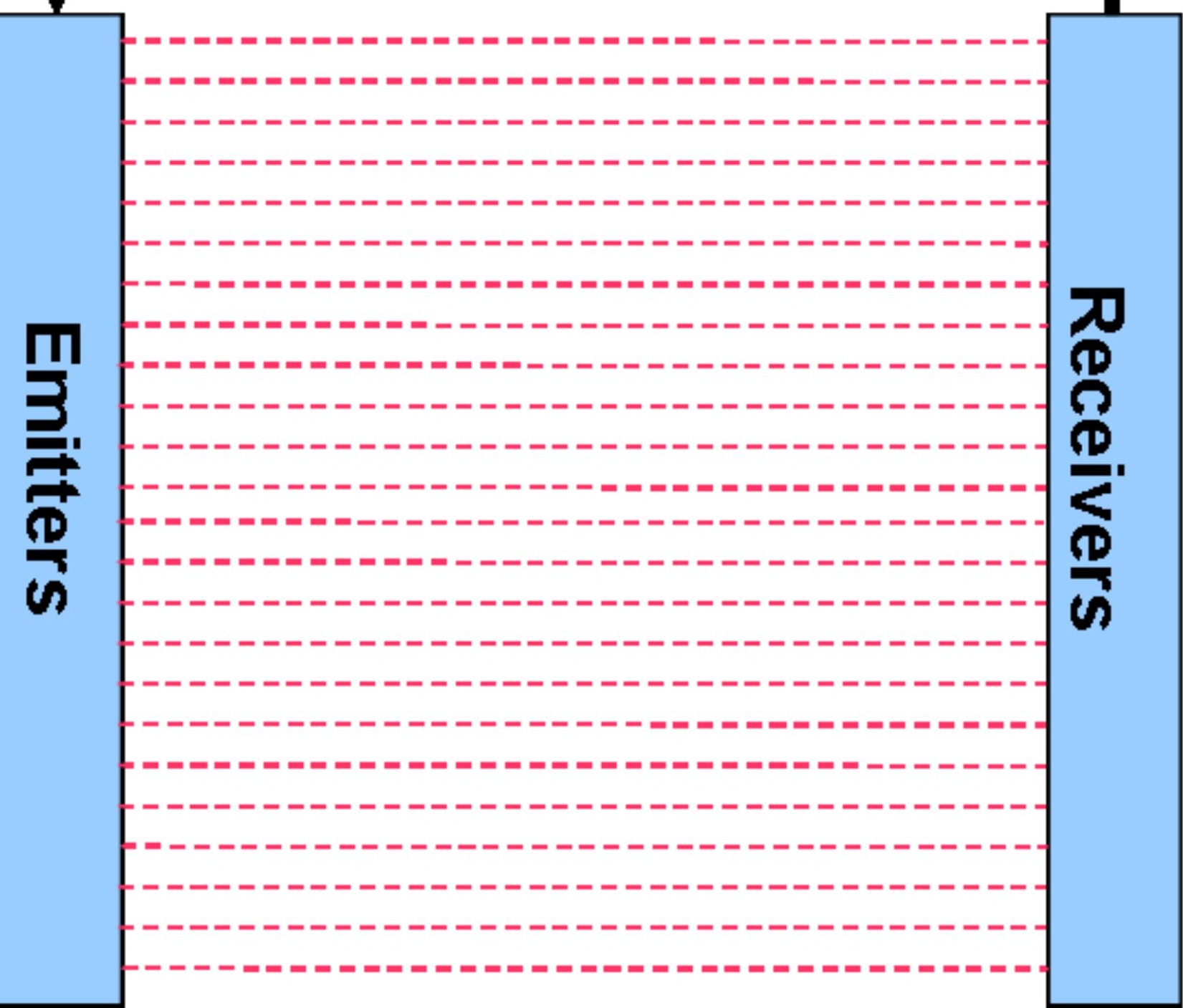



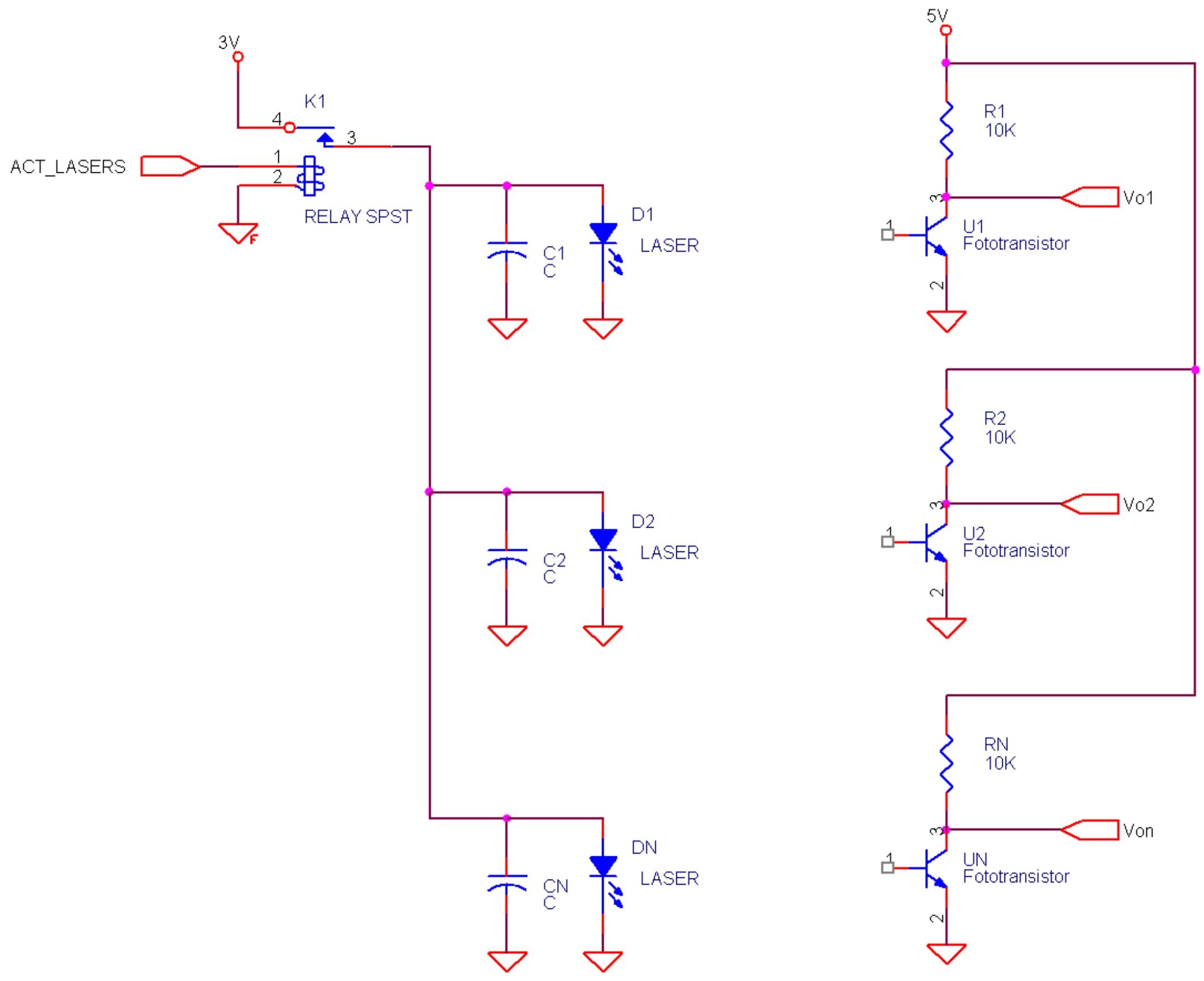


\section{Operaciones Acerca de...}

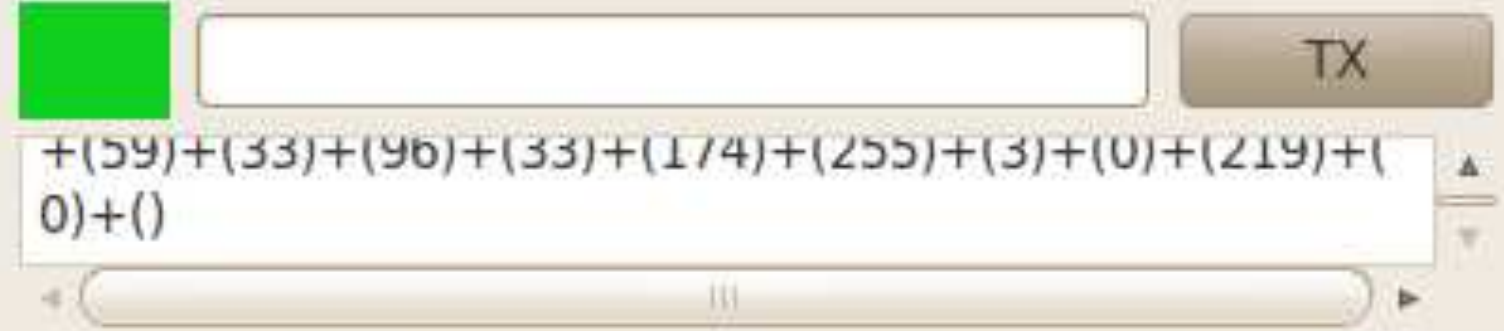

\section{Captura}
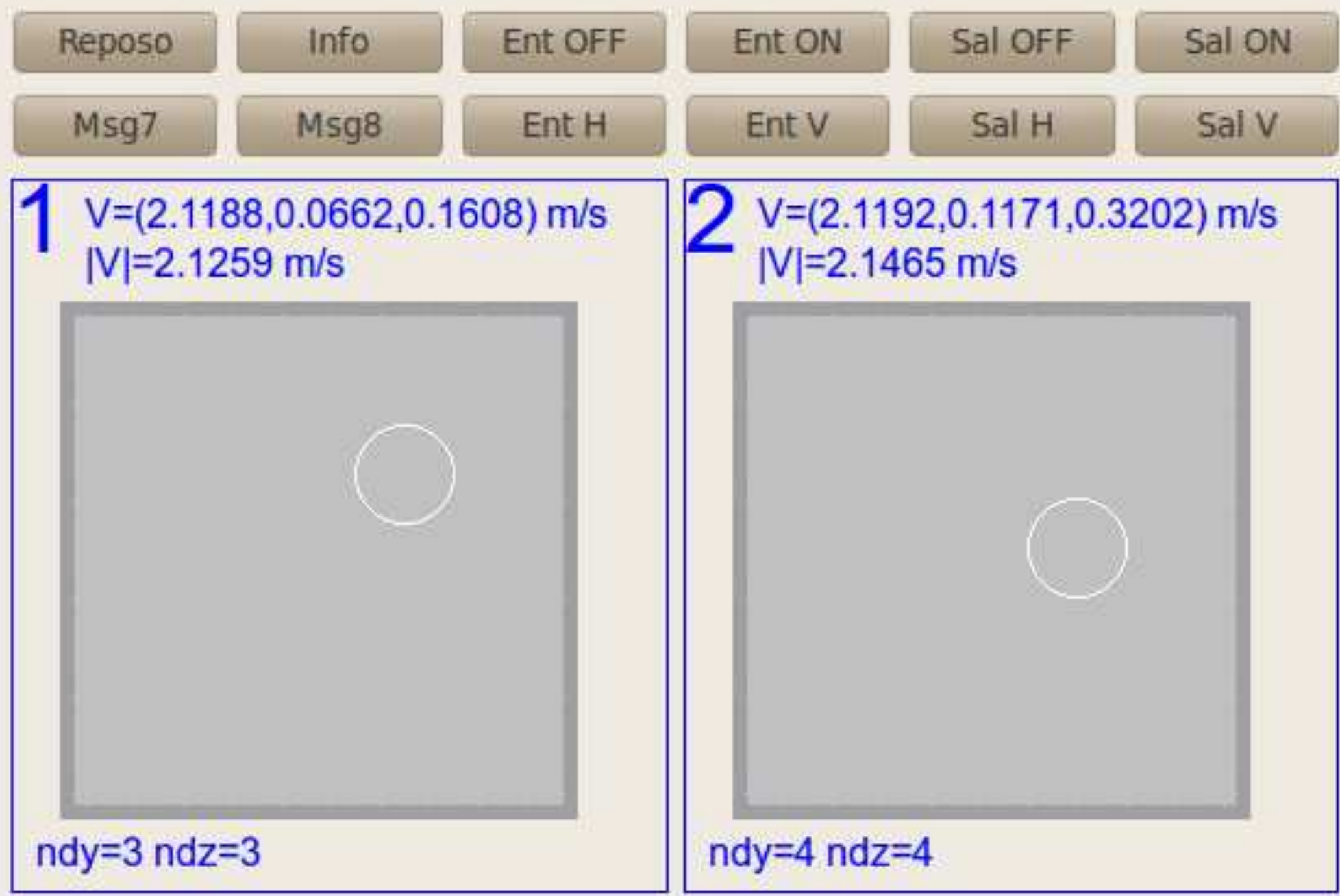

$\mathrm{V}=(2.1192,0.1171,0.3202) \mathrm{m} / \mathrm{s}$ $|\mathrm{V}|=2.1465 \mathrm{~m} / \mathrm{s}$

$n d y=4 n d z=4$ 
Fig. 1: Axis convention in an optical barrier.

Fig. 2: Detector activation geometry.

Fig. 3: Error caused by the gap between detectors.

Fig. 4: Diameter error in the worst case.

Fig. 5: Matlab code to calculate $\mathrm{v}$ and $\theta$.

Fig. 6: Optical barrier Hardware blocks diagram.

Fig. 7: Emitters and receivers block schematics.

Fig. 8: Control computer application. 